\title{
Male occult triple-negative breast cancer with dermatomyositis: a case report and review of the literature
}

This article was published in the following Dove Press journal:

OncoTargets and Therapy

14 November 2017

Number of times this article has been viewed

\author{
Le Zhang' \\ Chenghua Zhang ${ }^{2}$ \\ Zhaoying Yang' \\ Miao $\mathrm{He}^{3}$ \\ Lijuan Zhang' \\ Shereen Ezzat ${ }^{4}$ \\ Xi Liang ${ }^{5}$
}

'Department of Breast Surgery, China-Japan Union Hospital, Jilin

University, Changchun, Jilin, China;

${ }^{2}$ Endoscopy Department, Jilin Cancer

Hospital, Changchun, Jilin, China;

${ }^{3}$ Department of Anesthesia, The

Second Hospital of Jilin University,

Changchun, Jilin, China; ${ }^{4}$ Ontario

Cancer Institute and The Endocrine

Oncology Site Group, Princess

Margaret Hospital, University Health

Network, Toronto, Ontario, Canada;

${ }^{5}$ Department of Breast Surgery, The

Second Affiliated Hospital of Dalian

Medical University, Dalian, Liaoning,

China

Correspondence: Xi Liang

Department of Breast Surgery, The Second Affiliated Hospital of Dalian Medical University, 437 Zhongshan Road,

Sha hekou District., Dalian, Liaoning

I I602I, China

Tel/fax +86 4I| 8467 |29|

Email xiliangmed@I63.com

\begin{abstract}
Occult breast cancer is defined by the presence of axillary metastases without an identifiable primary breast tumor. Here, we report a rare case of a male occult breast cancer with dermatomyositis. We performed a modified radical mastectomy consisting of whole breast mastectomy and axillary lymph node dissection. Immunohistochemistry and fluorescent in situ hybridization analyses demonstrated an adenocarcinoma likely of breast origin, which was an occult triple-negative breast cancer. Interestingly, the patient's previously noted periorbital dermatomyositis resolved promptly following surgical excision.
\end{abstract}

Keywords: male breast cancer, occult breast cancer, triple-negative breast cancer, dermatomyositis

\section{Introduction}

Occult breast cancer $(\mathrm{OBC})$ is defined by the presence of axillary metastases without an identifiable breast tumor. It has been reported that most hormone receptor (HR)-positive rate of male breast cancer (MBC) is higher than that of female breast carcinomas. We encountered a unique case of $\mathrm{OBC}$, the immunohistochemistry (IHC) and fluorescent in situ hybridization (FISH) studies of which was a triple-negative breast carcinoma (TNBC). Interestingly, the patient developed periorbital erythematous papules 3 months before diagnosis of MBC. This was suspected to be dermatomyositis (DM), which has not been reported in male OBC patients previously. The purpose of this study is to describe and discuss the diagnosis, the clinicopathologic characteristics, and treatment of this rare subtype of breast carcinoma.

\section{Case report}

In May 2015, an 84-year-old male patient who denied any history of smoking or alcohol consumption, with no family history of breast cancer, discovered a palpable nodule in his right axilla. An ultrasound examination confirmed an irregular hypoechoic solid mass in the right axillary cavity measuring $3.9 \mathrm{~cm}$ in the longest diameter (Figure 1). Color Doppler signal demonstrated internal vascularity with absent fatty hilum. A core needle biopsy revealed a poorly differentiated adenocarcinoma (Figure 2), which was likely of breast origin. Three months before presentation to our department, the patient had developed periorbital erythematous papules, distributed over the lower eyelids, measuring $2.0 \times 1.5 \mathrm{~cm}$ (Figure 3 ), which was diagnosed as DM by the rheumatology department. Over the subsequent 3 months, he had also noted 


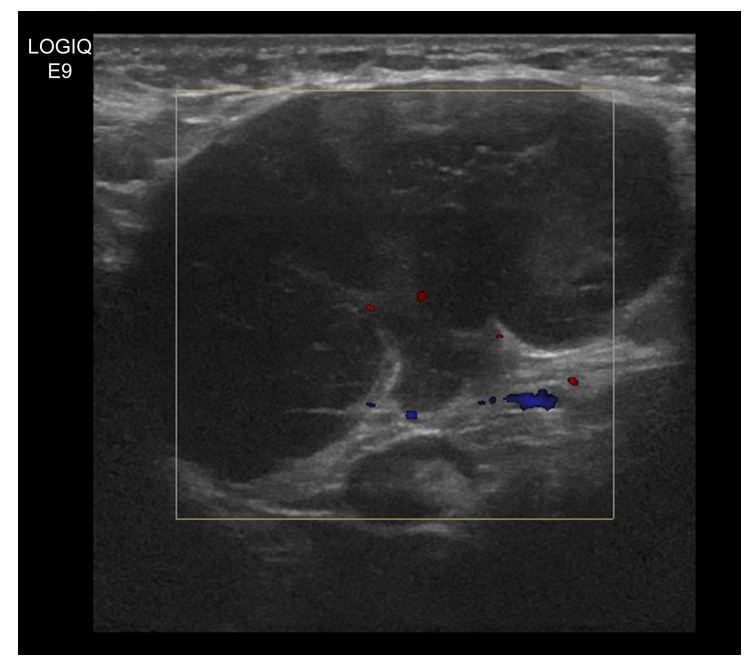

Figure I Ultrasound of the right axillary mass.

Notes: A low echo mass of $3.9 \times 2.9 \mathrm{~cm}$ can be seen in the right axillary mammary gland. The boundary is still clear and the shape is irregular. A strong blood flow signal can be detected within it.

progressive proximal upper muscle weakness in a symmetric distribution. However, he declined any therapeutic intervention.

Physical examination identified a nonmobile mass in the right axilla, measuring $5.0 \mathrm{~cm}$ in diameter; other superficial lymph nodes were not significantly enlarged and the skin covering the mass was normal. No nipple discharge and no masses were identified in either breast or other organs. A computed tomography scan of the brain, abdomen, and pelvis failed to reveal any malignant primary lesions. The bone scan screening for metastasis was negative.

On May 19, 2015, the patient underwent a modified radical mastectomy, which consisted of whole breast mastectomy and axillary lymph node dissection. However, no lesions were found on the right breast during surgery.

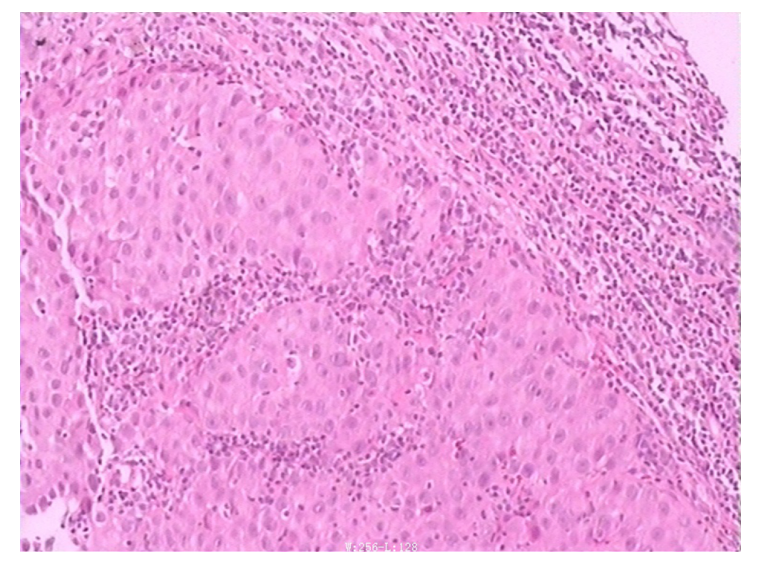

Figure 2 Image of core needle biopsy histologic diagnosis using hematoxylin and eosin staining (original $\times 100$ ).

Note: Metastasis of poorly differentiated carcinoma can be seen in the resected right axillary lymph node tissue.

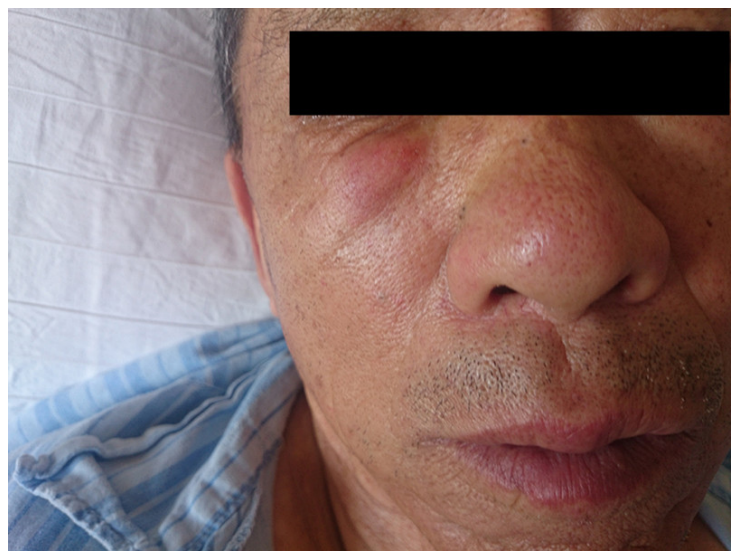

Figure 3 Preoperative facial photo of patient.

Note: Before surgery, the dermal erythematous papules on the right side of the lower eyelid are prominent.

The IHC of the lymph node tissue was negative for estrogen receptor (Figure 4A), progesterone receptor (Figure 4B), prostate-specific antigen (PSA) (-), positive gross cystic disease fluid protein-15 (GCDFP-15), AE1/AE3, cytokeratin 7 (CK7), CK20, and human epidermal growth factor receptor 2 (HER2), while HER2 was amplified (negative) by FISH analysis (Figure 4C). Dissection of axillary demonstrated 0 of 17 signs of metastatic lymph nodes except for the original malignant axillary lymph node. Based on these findings, he was diagnosed with right axillary metastatic TNBC presumably from an OBC. According to American Joint Committee on Cancer Staging, the stage of cancer classification was II stage $\left(\mathrm{T}_{0} \mathrm{~N}_{1} \mathrm{M}_{0}\right)$. The patient was submitted to adjuvant chemotherapy with 4 cycles of TC (Paclitaxel: $270 \mathrm{mg}$, Cyclophosphamide: $0.9 \mathrm{~g}$ ) and a rest of 21 days.

Following mastectomy, he experienced remarkable improvement of the dermal erythematous papules (Figure 5) and gradual recovery of muscle strength with normalization of creatinine kinase blood levels without glucocorticoid therapy.

Over $>2$ years of follow-up, the patient remained in good condition, without cutaneous or muscle DM recurrence or recurrence of breast cancer or other lesions.

\section{Discussion}

$\mathrm{OBC}$ is defined by the presence of axillary metastases without an identifiable breast tumor. It accounts for $0.3 \%-1 \%$ of all newly diagnosed malignant diseases of the breast. ${ }^{1}$ A man's lifetime risk of developing breast cancer is $\sim 0.7 \%$, but it has an increase in incidence in the seventh decade of life..$^{2,3}$ Because of the rarity of $\mathrm{OBC}$ in men, the diagnosis and treatment can be challenging. Most of the studies available in the literature have demonstrated that there were several reasons for palpable axillary masses, most commonly being 

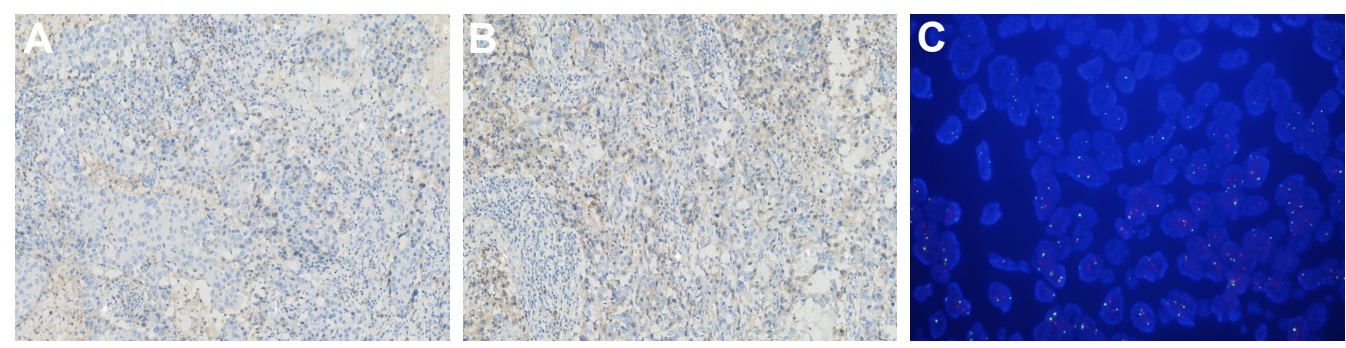

Figure 4 Expression of ER/PR by IHC staining (original $\times 100$ ) and HER2 by FISH analysis (original $\times 400$ ).

Notes: (A) ER-negative expression can be detected by IHC staining. (B) PR-negative expression can be detected by IHC staining. (C) HER2-negative expression result can be detected by FISH analysis.

Abbreviations: ER, estrogen receptor; PR, progesterone receptor; IHC, immunohistochemistry; HER2, human epidermal growth factor receptor 2; FISH, fluorescent in situ hybridization.

metastatic lymph nodes associated with breast cancer. ${ }^{4}$ When an axillary mass does occur, the most widely accepted method is to identify whether physical examination and imaging studies identify benign or malignant features. The next question is to confirm the primary source of disease.

Data can also be obtained for immunocytochemistry through a core needle biopsy of the metastasis. In this case, immunohistochemical and FISH studies revealed ER (-), PR (-), HER2 (-), GCDFP-15 (+), AE1/AE3 (+), CK7 (+), CK20 (+), and PSA (-). Thus, we diagnosed the axillary tumor as primary breast cancer with axillary lymph node involvement. Based on immunohistochemical and FISH analyses of ER, PR, Ki-67, and HER2, a simplified classification was adopted to identify different subtypes of breast cancer. In the previous literature, it has been reported that most primary lesions of $\mathrm{OBC}$ exhibit a significantly higher positive rate for hormone receptors than that of female breast carcinomas. ${ }^{5}$ Our case was even more unusual being TNBC.

TNBC, which is a special subtype of breast cancer, has been demonstrated to have a significantly higher risk of recurrence and death compared with luminal subtypes. ${ }^{6}$

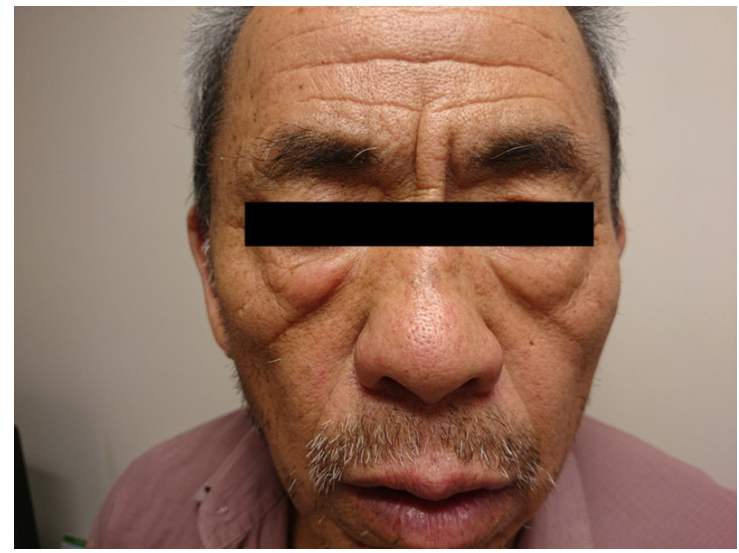

Figure 5 Postoperative facial photo of patient.

Note: Ten days after surgery, the dermal erythematous papules on the right side of the lower eyelid have nearly resolved.
Numerous studies have addressed the classification of TNBC to assist clinical management. Recently, Zhang et al described subnetwork biomarkers of low oncogenic GTPase activity, low ubiquitin/proteasome degradation, effective protection from oxidative damage, and tightly immune response to be linked with better prognosis through integrative analysis of cancer genomics data and protein interactome data. ${ }^{7}$ However, clinical data on TNBC in male patients are limited, and it is still unclear if the major prognostic factors in $\mathrm{MBC}$ are the same as those in women. An abstract presented at the 45th Annual Meeting of the American Society of Clinical Oncology in 2010 by Forester reported that male TNBC had a significantly worse prognosis than female TNBC or than other subtypes. ${ }^{8}$

Principles of treatment for male occult TNBC are similar to those of female breast cancer. Widely used adjuvant chemotherapy in breast cancer for TNBC is more chemosensitive than the HR-positive/HER2-negative phenotype. ${ }^{9}$ Compared with that of stage and subtype-matched patients, similar outcome was observed in OBC patients, while there was a trend for higher risk of recurrence and mortality in OBC patients with the triple negative subtype. ${ }^{10}$

DM is an idiopathic inflammatory myopathy associated with proximal muscle weakness and cutaneous findings such as heliotrope rashes, Gottron's papules, and periungual telangiectasis. ${ }^{11}$ Laboratory studies show creatine kinase elevation, electrophysiologic abnormalities, and inflammatory lesions on muscle biopsy. DM carries an increased risk of malignancy and can present as a paraneoplastic syndrome to multiple types of underlying malignancies, especially lung and prostate cancers, gastrointestinal tumors in men, and gynecological tumors in women, rarely in MBC. Polymyositis (PM)/DM can precede or present at the same time of or after the onset of malignant tumor development. Most commonly, however, the literature reports that malignant tumors appear $>2$ years after the onset of 
PM/DM. The risk of malignancy within the first year after the diagnosis of PM/DM is the highest, then decreases by each year, but remains higher than in the normal population. Therefore, the screening of malignant tumor should be carried out extensively within the first year after the appearance of PM/DM. Andras et al found that with 309 cases of PM/ DM analysis, ${ }^{12}$ among the 37 cases with malignant tumors, the proportion of malignancy and myositis in the first year was $64.8 \%$. Another study from 2003 to 2012, 246 cases of DM was retrospectively reviewed for 10 years. Including 60 cases with malignant tumors, 39 cases $(65.0 \%)$ occurred 1 year after diagnosis of DM. Malignancies were detected in $13(21.7 \%, 13 / 60)$ patients during the second year and 8 $(13.3 \%, 8 / 60)$ during the third year after DM. ${ }^{13}$

There is little evidence to guide treatment of DM. Wolff et al reported a rare case of DM with triple negative female breast cancer managed with chemotherapy and injectable adrenocorticotropic hormone agonist without surgery. ${ }^{14}$ However, the role of neoadjuvant chemotherapy is debatable. Ideally, detection and surgical removal of the malignant tumor yields the best results in improvement of DM. In this case, the patient developed periorbital erythematous papules 3 months before diagnosis of MBC. It is noteworthy that, after surgery, he experienced remarkable improvement in the dermal erythematous papules with gradual recovery of muscle strength without glucocorticoid therapy. These findings strongly confirm the importance of diagnosis and surgical treatment of the underlying malignancy.

The prognosis of DM patients with malignant tumors is poor. The main causes of death are extensive metastasis of tumor, secondary infection, and systemic failure. The overall survival rate of $\mathrm{PM} / \mathrm{DM}$ patients with malignant tumors is worse than that of other forms of myositis, and prognosis and life expectancy are determined by the underlying malignancies. ${ }^{15}$

\section{Conclusion}

We presented a rare case of male occult TNBC with DM. No conclusion has been drawn regarding current drug therapy, and surgery is still the main treatment.

OncoTargets and Therapy

\section{Publish your work in this journal}

OncoTargets and Therapy is an international, peer-reviewed, open access journal focusing on the pathological basis of all cancers, potential targets for therapy and treatment protocols employed to improve the management of cancer patients. The journal also focuses on the impact of management programs and new therapeutic agents and protocols on

Submit your manuscript here: http://www.dovepress.com/oncotargets-and-therapy-journal

\section{Acknowledgments}

Our study received approval from the ethics committee of the Second Affiliated Hospital of Dalian Medical University. The patient and family members also provided written informed consent to publish this case report details.

\section{Disclosure}

The authors report no conflicts of interest in this work.

\section{References}

1. Brill KL, Brenin DR. Occult breast cancer and axillary mass. Curr Treat Options Oncol. 2001;2(2):149-155.

2. He M, Liu H, Jiang Y. A case report of male occult breast cancer first manifesting as axillary lymph node metastasis with part of metastatic mucinous carcinoma. Medicine (Baltimore). 2015;94(25):e1038.

3. Khan MH, Allerton R, Pettit L. Hormone therapy for breast cancer in men. Clin Breast Cancer. 2015;15(4):245-250.

4. Park JE, Sohn YM, Kim EK. Sonographic findings of axillary masses: what can be imaged in this space? J Ultrasound Med. 2013;32(7): $1261-1270$.

5. Gu GL, Wang SL, Wei XM, Ren L, Zou FX. Axillary metastasis as the first manifestation of occult breast cancer in a male patient. Breast Care (Basel). 2009;4(1):43-45.

6. Chavez-Macgregor M, Clarke CA, Lichtensztajn D, Hortobagyi GN, Giordano SH. Male breast cancer according to tumor subtype and race: a population-based study. Cancer. 2013;119(9):1611-1617.

7. Zhang $\mathrm{F}$, Ren $\mathrm{C}$, Zhao $\mathrm{H}$, et al. Identification of novel prognostic indicators for triple-negative breast cancer patients through integrative analysis of cancer genomics data and protein interactome data. Oncotarget. 2016;7(44):71620-71634.

8. Arslan UY, Oksuzoglu B, Ozdemir N, et al. Outcome of non-metastatic male breast cancer: 118 patients. Med Oncol. 2012;29(2):554-560.

9. Yamamoto Y, Iwase H. Clinicopathological features and treatment strategy for triple-negative breast cancer. Int J Clin Oncol. 2010; 15(4):341-351.

10. Montagna E, Bagnardi V, Rotmensz N, et al. Immunohistochemically defined subtypes and outcome in occult breast carcinoma with axillary presentation. Breast Cancer Res Treat. 2011;129(3):867-875.

11. Yeh CN, Chen SC, Hwang TL, Chen MF, Liaw CC, Chan HL. Breast carcinoma in patients with dermatomyositis: a retrospective analysis of eight cases. Chang Gung Med J. 2002;25(6):374-380.

12. Andras C, Ponyi A, Constantin T, et al. Dermatomyositis and polymyositis associated with malignancy: a 21 -year retrospective study. J Rheumatol. 2008;35(3):438-444.

13. Chen D, Yuan S, Wu X, et al. Incidence and predictive factors for malignancies with dermatomyositis: a cohort from southern China. Clin Exp Rheumatol. 2014;32(5):615-621.

14. Wolff M, Mancuso C, Lal K, Dicostanzo D, Gropper C. Paraneoplastic dermatomyositis with cutaneous and myopathic disease responsive to adrenocorticotropic hormone therapy. J Clin Aesthet Dermato. 2017; 10(1):57-62.

15. Ponyi A, Constantin T, Garami M, et al. Cancer-associated myositis: clinical features and prognostic signs. Ann N Y Acad Sci. 2005;1051:64-71.

patient perspectives such as quality of life, adherence and satisfaction. The manuscript management system is completely online and includes a very quick and fair peer-review system, which is all easy to use. Visit http://www.dovepress.com/testimonials.php to read real quotes from published authors. 